Original Article

\title{
The Effect of Moral Disengagement on Bystander's Behavior in Bullying Among Middle School Students
}

\author{
Nguyen Le Que An ${ }^{1, *}$, Nguyen Thi My Linh ${ }^{1}$, Bui Thi Diem $\mathrm{My}^{2}$, \\ Tu Minh Phuong ${ }^{1}$ Tran Thi Le Thu ${ }^{1}$, Pham Thi Dieu Thuy ${ }^{1}$ \\ ${ }^{I}$ Department of Psychology and Education, Hanoi University of Education, \\ 136 Xuan Thuy, Cau Giay District, Hanoi, Vietnam \\ ${ }^{2}$ Nguyen Binh Khiem Secondary and High School, 6 Tran Quoc Hoan, \\ Cau Giay, Hanoi, Vietnam
}

Received 11 June 2021

Revised 13 July 2021; Accepted 29 July 2021

\begin{abstract}
The aim of the current study was to examine use of moral disengagement among middle school students as bystanders to bullying, as well as the links between four domains of moral disengagement and bystander's behaviors in bullying episode, including pro-bullying, victim-defending and passive bystanding behavior. Participants included 736 adolescents from the urban area of Hanoi $\left(M_{\text {age }}=13.69 ; 53.1 \%\right.$ male $)$. According to the main hypotheses, all four domains of moral disengagement were negatively correlated with victim-defending behavior and positively correlated with passive bystanding behavior, whereas only three sets of mechanisms were found to be related to pro-bullying behavior, including minimizing one's agentive role, consequences distortion, and victim attribution. Linear regression analyses indicated that bystander's behaviors were predicted by minimizing one's agentive role. Differences regarding gender and grade were also found. These results highlight the importance of particular moral disengagement sets of mechanisms and have potential implications for prevention tackling bullying.
\end{abstract}

Keywords: Shool bullying, bystander, moral disengagement, defending behavior, pro-bullying behavior, middle school.

\footnotetext{
${ }^{*}$ Corresponding author.

E-mail address: ann.ngnle@gmail.com

https://doi.org/10.25073/2588-1159/vnuer.4539
} 


\title{
Ảnh hưởng của buông lỏng đạo đức tới hành vi của bên chứng kiến trong bắt nạt ở học sinh trung học cơ sở
}

\author{
Nguyễn Lê Quế An ${ }^{1, *}$, Nguyễn Thị Mỹ Linh ${ }^{1}$, Bùi Thị Diễm My², \\ Từ Minh Phương ${ }^{1}$, Trần Thị Lệ Thu ${ }^{1}$, Phạm Thị Diệu Thúy ${ }^{1}$, \\ ${ }^{1}$ Truòng Đại học Su phạm Hà Nội, 136 Xuân Thủy, Cà̀u Giấy, Hà Nội, Việt Nam \\ ${ }^{2}$ Trường Trung hoc Co sở và Trung họ Phổ thông Nguyễn Bỉnh Khiêm, \\ 6 Trần Quốc Hoàn, Cầu Giấy, Hà Nội, Việt Nam \\ Nhận ngày 11 tháng 6 năm 2021 \\ Chỉnh sửa ngày 13 tháng 7 năm 2021; Chấp nhận đăng ngày 29 tháng 7 năm 2021
}

\begin{abstract}
Tóm tắt: Nghiên cứu hiện tại được tiến hành nhằm làm rõ thực trạng buông lỏng đạo đức trong bắt nạt của học sinh chứng kiến, đồng thời khám phá mối quan hệ giữa bốn khu vực cơ chế buông lỏng đạo đức và ba dạng biểu hiện hành vi của cá nhân với tư cách là bên chứng kiến trong bắt nạt, bao gồm biểu hiện hành vi bảo vện nạn nhân, biểu hiện hành vi củng cố bắt nạt và biểu hiện hành vi không can dự. Kết quả nghiên cứu trên khách thể học sinh trung học cơ sở $\mathrm{N}=736\left(M_{\text {tuó̉i }}=13,69\right.$; $53,1 \%$ nam) cho thấy khu vực tái cấu trúc hành vi gây hại là khu vực cơ chế được học sinh sử dụng nhiều nhất, với sự khác biệt giữa hai giới và các khối lớp trong một số khu vực cơ chế. Kết quả nghiên cứu cho thấy cả bốn khu vực cơ chế của buông lỏng đạo đức đều có tương quan nghịch với hành vi bảo vệ nạn nhân, tương quan thuận với hành vi không can dự; trong khi chỉ có ba khu vực cơ chế, bao gồm làm giảm trách nhiệm cá nhân, bóp méo hậu quả và quy kết trách nhiệm cho nạn nhân, có tương quan thuận với hành vi củng cố bắt nạt. Phẩn tích hồi quy tuyến tính đơn biến cho thấy khu vực cơ chế làm giảm trách nhiệm cá nhân có khả năng dự báo ba dạng biểu hiện hành vi của bên chứng kiến. Khuyển nghị cho công tác phòng ngừa bắt nạt học đường dựa trên kết quả nghiên cứu lý luận và thực tiễn cũng sẽ được bàn luận trong bài viết này.
\end{abstract}

Từ khóa: Bắt nạt học đường, bên chứng kiến, buông lỏng đạo đức, hành vi bảo vệ, hành vi củng cố bắt nạt, trung học cơ sở.

\section{Mở đầu}

Bắt nạt học đường có thể được xem là một trong những vấn đề nghiêm trọng, đe dọa trực tiếp tới sự an toàn của trường học, với hậu quả lâu dài để lại trên học sinh. Bắt nạt có thể được định nghĩa là hệ thống hành vi có chủ đích gây hại, được thực hiện nhiều lần bởi một hoặc nhiều học sinh; với sự chênh lệch về mặt sức mạnh giữa học sinh bắt nạt và học sinh nạn nhân (Olweus, 1994) [1]. Nhằm làm rõ hơn về bản chất và sự kéo dài của hiện tượng này, vị trí và vai trò của bộ phận học sinh chứng kiến - là bộ phận chiếm đa số trong vụ bắt nạt (Craig và

\footnotetext{
* Tác giả liên hệ.

Địa chỉ email: ann.ngnle@ gmail.com

https://doi.org/10.25073/2588-1159/vnuer.4539
}

cộng sự., 2000; Hawkins và cộng sự, 2001; O’Connell và cộng sự., 1999; Rigby \& Johnson, 2006) [2-5], đã được nhìn nhận và xem xét là có ý nghĩa lớn với công tác phòng ngừa (O'Connell và cộng sự, 1999; Salmivalli và cộng sự, 1996; Salmivalli và Voeten, 2004) $[4,6,7]$. Bên chứng kiến trong bắt nạt, dù không trực tiếp khởi xướng hoặc thực hiện hành vi bắt nạt, nhưng có khả năng thay đổi cục diện vụ bắt nạt theo những chiều hướng khác nhau (Salmivalli, 1999; Salmivalli, 2014) [8, 9]. Như vậy, việc nâng cao, khuyến khích học sinh thực hiện hành vi bảo vệ nạn nhân (ví dụ: hỗ trợ nạn nhân tìm kiếm sự giúp đỡ, làm bạn với nạn nhân, báo cho người lớn) cùng với làm giảm lựa chọn hành vi củng cố bắt nạt (ví dụ: cùng tham gia bắt nạt nạn nhân, cổ vũ bắt nạt, ngăn cản học sinh tìm sự trợ giúp) của học sinh 
chứng kiến cần được xem là một trong những mục tiêu của các chương trình phòng ngừa - can thiệp bắt nạt (Vannini và cộng sự, 2011) [10]. Đồng thời, điều này đặt ra nhu cầu nghiên cứu, khám phá những nhân tố tác động, chi phối tới lựa chọn hành vi của bên chứng kiến. Cùng với mục đích khám phá lý do vì sao trẻ em và thanh thiếu niên tham gia vào việc củng cố, cổ vũ bắt nạt và từ chối giúp đỡ nạn nhân ngay cả khi hầu hết các em đều đánh giá bắt nạt là hành động sai và có tính chất gây hại (Thornberg, 2010) [11]; nghiên cứu trong khoảng hai thập kỷ trở lại đây đã tập trung vào quá trình nhận thức đạo đức của học sinh nói chung và buông lỏng đạo đức nói riêng, cũng như mối quan hệ của những biến này với hành vi gây hấn, bắt nạt và hành vi của bên chứng kiến (Barchia và Bussey, 2011; Bjärehead và cộng sự, 2020; Gini, 2006; Hymel và cộng sự, 2005; Thornberg và Jungert, 2013, 2014; Thornberg và cộng sự, 2017) [12-18].

Tại Việt Nam, trong khoảng hơn một thập kỷ trở lại đây, bắt nạt học đường đã được nhiều nhà nghiên cứu quan tâm. Trong những nghiên cứu gần đây, bắt nạt thường được phân loại thành hai dạng chính là bắt nạt truyền thống (bắt nạt thể chất, bắt nạt mối quan hệ, bắt nạt lời nói, xâm phạm tài sản) và bắt nạt trực tuyến (xem Trần Văn Công, 2017, 2018; Trần Văn Công và cộng sự, 2009) [19-21]. Đồng thời, ảnh hưởng của những yếu tố như giới tính, độ tuổi, tình trạng sức khỏe tâm thần, phản ứng từ bạn bè, chất lượng tình bạn, thành tích học tập của cá nhân (xem Lê và cộng sự, 2017; Phạm Thị Thu Ba và Trần Quỳnh Anh, 2016; Trần Văn Công và Nguyễn Thị Hoài Phương, 2018; Trần và cộng sự, 2020) [22-25] tới mức độ học sinh tham gia vào vụ bắt nạt và bị bắt nạt trong các dạng bắt nạt trên cũng được chứng minh. Tuy nhiên, trong phạm vi tìm kiếm của chúng tôi, hầu hết các nghiên cứu về bắt nạt học đường tại Việt Nam tới thời điểm này chủ yếu tập trung vào học sinh bắt nạt và học sinh nạn nhân, chưa chú ý nhiều tới bên chứng kiến như một bộ phận có thể tác động tới bắt nạt. Bên chứng kiến, dù đã được đề cập ở một vài nghiên cứu đi trước (xem Lê và cộng sự, 2017; Trần và cộng sự, 2018) [22,26] nhưng các nghiên cứu về đặc điểm, yếu tố tác động tới bộ phận này hiện vẫn còn chưa nhiều.

\section{Buông lỏng đạo đúc}

Trong thuyết nhận thức xã hội (Bandura và cộng sự, 1996; Bandura, 1999; Bandura, 2002) [27-29], buông lỏng đạo đức được định nghĩa là một quá trình nhận thức xã hội cho phép cá nhân nới lỏng các chuẩn mực đạo đức nhằm thực hiện những hành vi có chủ đích gây hại, hành vi phi đạo đức và hợp thức hóa những hành vi đó là phù hợp với chuẩn mực và quy tắc đạo đức của bản thân và cộng đồng. Theo Bandura $(1986,2014)[30,31]$, con người được xem là những chủ thể tích cực theo đuổi những mục tiêu phù hợp với giá trị của bản thân, kiểm soát và ra quyết định dựa trên chuẩn mực đạo đức của cá nhân trong các tình huống xã hội, điều chỉnh hành vi của bản thân bằng việc cân nhắc những hệ quả có thể xảy ra. Tuy nhiên, khi quá trình buông lỏng đạo đức diễn ra, những cơ chế tự kiểm soát và điều chỉnh hành vi thông thường sẽ bị vô hiệu hóa, từ đó cho phép cá nhân thực hiện những hành vi gây hại và vẫn có thể duy trì, giữ vững được quy tắc đạo đức thông thường (Bandura, 1999) [28]. Những hành vi có chủ đích gây hại, sau khi được biện hộ và hợp thức hóa sẽ trở nên phù hợp với chuẩn mực đạo đức thông thường, và cá nhân có thể sẽ không trải nghiệm những cảm xúc như tội lỗi, ân hận. Cụ thể, quá trình này diễn ra theo tám cơ chế, thuộc bốn khu vực cơ chế chủ yếu, bao gồm: i) Tái cấu trúc hành vi gây hại (biện hộ đạo đức, sử dụng ngôn ngữ nói giảm, nói tránh, so sánh giảm nhẹ); ii) Làm mờ/làm giảm trách nhiệm cá nhân (chia mỏng trách nhiệm, di chuyển trách nhiệm); iii) Diễn giải sai lệch/bóp méo hậu quả (diễn giải sai lệch hậu quả của hành vi gây hại); và iv) Quy kết trách nhiệm (Bandura và cộng sự, 1996; Bandura, 2002) $[27,29]$. Những cơ chế thuộc khu vực tái cấu trúc hành vi gây hại bao gồm những cơ chế cho phép cá nhân biện hộ đạo đức với hành vi gây hại của mình là hành vi chính nghĩa cho một mục đích cao đẹp, đúng đắn; so sánh hành vi có tính chất phương hại của mình với những hành vi nguy hại, khủng khiếp hơn nhằm giảm nhẹ mức độ nghiêm trọng thuộc tính chất hành 
vi; hoặc diễn giải và trình bày về hành vi gây phương hại bằng uyển ngữ, từ đó làm giảm tính chất gây hại và nghiêm trọng của hành vi. Khu vực làm mờ/làm giảm trách nhiệm bao gồm những cơ chế cho phép cá nhân di chuyển trách nhiệm của bản thân với hành vi gây hại lên những đối tượng khác (xem căn nguyên của việc vi phạm đạo đức là xuất phát từ áp lực tình thế hoặc do yêu cầu, chỉ thị của người khác); hoặc chia mỏng trách nhiệm của cá nhân với những cá nhân liên quan khác. Khu vực cơ chế tập trung vào hậu quả của hành vi gây hại cho phép cá nhân bóp méo hoặc phủ nhận hậu quả tiêu cực của hành vi. Theo Bandura (1999) [28], hậu quả của hành động làm hại người khác càng khó nhìn thấy, cũng như việc hành động mang tính chất gây hại bị tách rời ra khỏi hậu quả thật của nó, thì việc thực hiện hành vi làm hại người khác càng dễ dàng diễn ra. Khu vực cơ chế tập trung vào nạn nhân của hành vi gây hại bao gồm những cơ chế cho phép cá nhân tước bỏ những đặc điểm con người của nạn nhân, phi nhân hóa nạn nhân hoặc quy kết trách nhiệm cho nạn nhân, lập luận rằng hành vi vi phạm chuẩn mực đạo đức của mình là phản ứng tất yếu hoặc đáp trả trước sự khiêu khích (đến từ phía nạn nhân); nạn nhân xứng đáng và/hoặc phải tự chịu trách nhiệm với hậu quả tiêu cực xảy ra với họ (Bandura, 2016) [32]. Quy kết trách nhiệm thường được sử dụng là công cụ để hợp thức hóa hành vi của thủ phạm trong rất nhiều tình huống, bối cảnh khác nhau, ví dụ như khi một học sinh bắt nạt người khác cho rằng việc làm của mình là phản ứng tất yếu trước sự khiêu khích trước đó của nạn nhân (Boulton và Underwood, 1992) [33].

Trong nghiên cứu về hành vi gây hấn của thanh thiếu niên, buông lỏng đạo đức có thể được xem là một yếu tố nguy cơ khi trẻ em và thanh thiếu niên với mức độ buông lỏng đạo đức cao có xu hướng thể hiện, thực hiện hành vi gây hấn cao hơn so với bạn đồng trang (Bandura và cộng sự, 1996; Pornari và Wood, 2010) [27, 34], bao gồm cả hành vi bắt nạt và ủng hộ bắt nạt (Perren và GutzwillerHelfenfinger, 2012; Thornberg và Jungert, 2013) $[16,35]$.
Buông lỏng đạo đúc và hành vi của bên
chứng kiến trong bắt nạt
Từ giả thiết về mối quan hệ giữa buông lỏng đạo đức và hành vi gây hấn (Hymel và cộng sự, 2005) [15], hành vi thuận xã hội của cá nhân (Bandura và cộng sự, 1996) [27], mối quan hệ giữa buông lỏng đạo đức và hành vi của bên chứng kiến trong bắt nạt cũng đã được các nhà nghiên cứu chú ý trong những năm trở lại đây (Bussey và cộng sự, 2020; Caravita và cộng sự., 2014; Gini và cộng sự, 2015; Obermann, 2011) [36-39]. Kết quả nghiên cứu về những mối quan hệ này có tương đồng với khung lý thuyết của buông lỏng đạo đức (Bandura, 1999) [28], trẻ em và thanh thiếu niên có xu hướng thực hiện hành vi bảo vệ nạn nhân bắt nạt thể hiện mức độ buông lỏng đạo đức thấp hơn so với học sinh bắt nạt (Bussey và cộng sự, 2020; Caravita và cộng sự, 2012; Gini, 2006) [36, 40, 14]. Ngược lại, hành vi không can dự của học sinh chứng kiến cho thấy tương quan thuận với buông lỏng đạo đức (Obermann, 2011) [39]. Tuy nhiên, những nghiên cứu thực tiễn về mối quan hệ của từng cơ chế và nhóm cơ chế cụ thể của buông lỏng đạo đức với biểu hiện hành vi của bên chứng kiến vẫn còn chưa nhiều và chưa thống nhất. Trong nghiên cứu này, chúng tôi sẽ tiến hành xem xét mối quan hệ giữa bốn khu vực cơ chế của quá trình buông lỏng đạo đức với ba dạng biểu hiện hành vi của bên chứng kiến trong bắt nạt, bao gồm hành vi bảo vệ nạn nhân, hành vi củng cố bắt nạt và hành vi không can dự.

\section{Tổ chức và phương pháp nghiên cứu}

\subsection{Mẫu nghiên cứu}

Nghiên cứu này sử dụng phương pháp chọn mẫu tiện lợi. Khảo sát được tiến hành trên khách thể 736 học $\sinh \left(M_{\text {Tuổi }}=13,69\right.$; $S D_{\text {Tuổi }}=1,146 ; 53,1 \%$ nam) từ 32 lớp của 05 trường THCS thuộc nội thành Hà Nội. Quá trình khảo sát được thực hiện với sự hỗ trợ của Ban giám hiệu nhà trường và các giáo viên chủ nhiệm. 


\subsection{Công cu đo lường}

2.2.1. Thang đo Buông lỏng đạo đức của bên chứng kiến trong bắt nạt học đường

Thang đo Buông lỏng đạo đức của bên chứng kiến trong bắt nạt học đường gồm 12 item, được thiết kế nhằm đo lường quá trình buông lỏng đạo đức của học sinh chứng kiến trong bắt nạt theo bốn khu vực cơ chế, bao gồm: tái cấu trúc hành vi gây hại (ví dụ: " $K h i$ có bắt nạt, em muốn thể hiện sự phản đối của mình bằng cách tránh $x a$, không tham gia vào"), làm giảm trách nhiệm cá nhân (ví dụ: "Khi mọt vu bắt nạt diễn ra trong lóp, em không cần báo cho cô giáo biết vì các bạn khác sẽ báo lại cho cô”'), phủ nhận/bóp méo hậu quả (ví dụ: "Việc bịa chuyện về một bạn trong lớp chi là môt trò đùa vui vô hại”), quy kết trách nhiệm cho nạn nhân (ví dụ, "Nếu một bạn bi bắt nạt thì chắc hẳn bạn ấy đã làm gì sai truớc đó"); sử dụng Likert 5 (1 = "rất không đồng y"; 5 = "rất đồng $y$ "). Thang đo ban đầu được xây dựng bao gồm 27 item. Chúng tôi đã phỏng vấn 19 học sinh THCS nhằm kiểm tra và điều chỉnh cách diễn đạt của các item. Sau khi điều chỉnh, các thuộc tính đo lường của thang được kiểm định trên khách thể học sinh THCS $(\mathrm{N}=188$; $M_{\text {Tuổi }}=12,76 ; S D_{\text {Tuổi }}=0,76$ ) với độ tin cậy của từng khu vực cơ chế được đảm bảo: tái cấu trúc hành vi gây hại $(\alpha=0,71)$, làm giảm trách nhiệm cá nhân $(\alpha=0,66)$, phủ nhận/bóp méo hậu quả $(\alpha=0,78)$, quy kêt trách nhiệm cho nạn nhân $(\alpha=0,61)$. Kết quả phân tích nhân tố khám phá EFA (PCA, Varimax, $f l=0,45$ ) cho thấy 12 item được giữ lại, có độ hiệu lực cấu trúc được đảm bảo với hệ số $\mathrm{KMO}=0,79$; Bartlett's test $\chi^{2}(66)=424,04, p<0,05$; 4 nhân tố được trích xuất có trị số Eigenvalue nằm trong khoảng từ 0,98 đến 3,53 ; tổng phần trăm phương sai trích $=58,72 \%$. Kết quả khảo sát trên khách thể 736 học sinh, thang đo đáp ứng được yêu cầu về độ tin cậy, cụ thể: tái cấu trúc hành vi gây hại $(\alpha=0,72)$, làm giảm trách nhiệm cá nhân $(\alpha=0,66)$, phủ nhận/bóp méo hậu quả $(\alpha=0,78)$, quy kết trách nhiệm cho nạn nhân $(\alpha=0,68)$.
2.2.2. Thang đo Biểu hiện tác động của bên chứng kiến trong bắt nạt học đường

Thang đo Biểu hiện tác động của bên chứng kiến trong bắt nạt học đường được xây dựng nhằm đo lường các biểu hiện tác động tới vụ bắt nạt của bên chứng kiến. Biểu hiện tác động của bên chứng kiến là những lựa chọn hành vi cụ thể, điển hình của học sinh khi chứng kiến bắt nạt, có thể thay đổi cục diện vụ bắt nạt theo những chiều hướng khác nhau, được xét theo ba dạng biểu hiện: bảo vệ nạn nhân (ví dụ: "Nếu trong lớp có bạn bị tẩy chay, em sẽ báo cho giáo viên hoặc người lớn"), củng cố bắt nạt (ví dụ: "Khi thấy một bạn đang bị đánh, em sẽ đúng xem và cổ vü"), và không can dự (ví dụ: "Khi một bạn trong lớp bị nói xấu, em sẽ lờ đi và coi nhu không nghe thấy"); sử dụng thang Likert 5 (1 = "không bao giờ"; 5 = "luôn luôn"). Thang đo ban đầu được xây dựng bao gồm 22 item. Chúng tôiđã phỏng vấn 19 học sinh nhằm kiểm tra và điều chỉnh cách diễn đạt của các item. Sau khi điều chỉnh, những thuộc tính đo lường của thang được kiểm định trên 203 học sinh THCS ( $M_{\text {Tuổi }}=12,74 ; S D_{\text {Tuổi }}=0,77 ; 52,7 \%$ nam). Độ tin cậy của các tiểu thang, bao gồm bảo vệ nạn nhân $(\alpha=0,83)$, củng cố bắt nạt $(\alpha=0,76)$, và không can dự $(\alpha=0,78)$ được đảm bảo. Kết quả phân tích EFA (PCA, Varimax, $f l=0,5)$ cho thấy 14 items được giữ lại có độ hiệu lực cấu trúc được đảm bảo với hệ số $\mathrm{KMO}=$ 0,85 ; Bartlett's test $\chi^{2}(91)=745,16$, $p<0,05 ; 3$ nhân tố được trích xuất có trị số Eigenvalue $>1$; tổng phương sai trích $=54,46 \%$. Kết quả khảo sát khách thể 736 học sinh cho thấy thang đo đáp ứng được yêu cầu về độ tin cậy, cụ thể: bảo vệ nạn nhân $(\alpha=0,82)$, củng cố bắt nạt $(\alpha=0,61)$, và không can dự $(\alpha=0,79)$.

\section{3. Đạo đức nghiên cúu}

Khảo sát được thực hiện với sự cho phép của 05 trường THCS thuộc nội thành Hà Nội. Quy trình khảo sát đảm bảo tính khách quan. Mọi thông tin và dữ liệu thu được từ quá trình khảo sát đều được bảo mật và chỉ công bố ẩn danh. Dữ liệu và kết quả nghiên cứu chỉ được sử dụng cho mục đích khoa học và không phục vụ cho bất kỳ mục đích nào khác. 


\section{Kết quả nghiên cứu}

\subsection{Các kết quả mô tả ban đầu}

Kết quả cho thấy khu vực cơ chế buông lỏng đạo đức trong bắt nạt đạt mức điểm trung bình cao nhất là khu vực cơ chế tái cấu trúc hành vi gây hại $(M=2,97 ; S D=0,99)$, khu vực cơ chế đạt mức điểm trung bình thấp nhất là khu vực cơ chế phủ nhận/bóp méo hậu quả $(M=1,86 ; S D=0,97)$. Có sự khác biệt giữa hai giới trong khu vực cơ chế phủ nhận/bóp méo hậu quả, với học sinh nam $(M=1,95$; $S D=0,96$ ) thể hiện rõ hơn học sinh nữ $(M=1,76 ; S D=0,95)\left(t_{729}=2,78 ; p<0,05\right)$. Phân tích sâu ANOVA một yếu tô được sử dụng nhằm làm rõ sự khác biệt giữa các khối lớp ở các khu vực cơ chế buông lỏng đạo đức trong bắt nạt. Kết quả phân tích cho thấy học sinh khối 8 thể hiện rõ hơn học sinh khối 9 ở khu vực cơ chế quy kết trách nhiệm cho nạn nhân $\left(F_{3 ; 732}=3,02 ; p<0,05\right)$. Học sinh khối 6 thể hiện ít hơn học sinh ba khối còn lại ở hai khu vực cơ chế: phủ nhận/bóp méo hậu quả $\left(F_{3 ; 336,72}=13,88 ; p<0,05\right)$ và làm giảm trách nhiệm cá nhân $\left(F_{3 ; 331,53}=16,80 ; p<0,05\right)$.
Đồng thời, học sinh khối 8 thể hiện rõ hơn học sinh khối 7 và khối 9 ở khu vực cơ chế làm giảm trách nhiệm cá nhân $\left(F_{3 ;} 331,53=16,80\right.$; $p<0,05)$.

3.2. Mối quan hệ giũa buông lỏng đạo đức và hành vi của bên chứng kiến trong bắt nạt

Tương quan giữa bốn khu vực cơ chế buông lỏng đạo đức và ba dạng biểu hiện hành vi của bên chứng kiến trong bắt nạt được xem xét sử dụng hệ số tương quan Pearson (Bảng 1). Như kỳ vọng ban đầu, tất cả khu vực cơ chế của quá trình buông lỏng đạo đức có tương quan nghịch với hành vi bảo vệ nạn nhân $(p<0,01)$; có tương quan thuận với lựa chọn hành vi không can dự $(p<0,01)$ của học sinh khi chứng kiến bắt nạt. Đối với hành vi củng cố bắt nạt, có tồn tại mối tương quan thuận với ba khu vực cơ chế: làm giảm trách nhiệm cá nhân $(r=0,40$; $p<0,01)$, phủ nhận/bóp méo hậu quả $(r=0,31$; $p<0,01)$, và quy kết trách nhiệm cho nạn nhân $(r=0,19 ; p<0,01)$. Trong đó, có thể thấy khu vực cơ chế làm giảm trách nhiệm cá nhân có tương quan mạnh nhất với cả ba dạng hành vi của bên chứng kiến (Bảng 1).

Bảng 1. Tương quan giữa khu vực cơ chế buông lỏng đạo đức với biểu hiện tác động của bên chứng kiến

\begin{tabular}{|l|c|c|c|c|}
\hline & $\begin{array}{c}\text { Tái cấu trúc } \\
\text { hành vi gây hại }\end{array}$ & $\begin{array}{c}\text { Làm giảm } \\
\text { trách nhiệm cá nhân }\end{array}$ & $\begin{array}{c}\text { Phủ nhận hoặc } \\
\text { bóp méo hậu quả }\end{array}$ & $\begin{array}{c}\text { Quy kết trách } \\
\text { nhiệm cho nạn nhân }\end{array}$ \\
\hline Bảo vệ nạn nhân & $-0,19^{* *}$ & $-0,44^{* *}$ & $-0,21^{* *}$ & $-0,27^{* *}$ \\
\hline Củng cố bắt nạt & 0,05 & $0,40^{* *}$ & $0,31^{* *}$ & $0,19^{* *}$ \\
\hline Không can dự & $0,32^{* *}$ & $0,38^{* *}$ & $0,16^{* *}$ & $0,23^{* *}$ \\
\hline
\end{tabular}

Ghi chú: ** Tương quan có ý nghĩa thống kê ở mức 0,01 (2 đuôi).

\subsection{Phân tích hồi quy}

Phân tích hồi quy tuyến tính đơn biến được sử dụng nhằm làm rõ ảnh hưởng và khả năng dự báo của khu vực cơ chế làm giảm trách nhiệm cá nhân tới các dạng biểu hiện hành vi của học sinh khi chứng kiến bắt nạt: bảo vệ nạn nhân, củng cố bắt nạt, và không can dự (Bảng 2).

Hành vi bảo vệ nạn nhân: Kết quả phân tích hồi quy tuyến tính với khu vực cơ chế làm giảm trách nhiệm cá nhân là biến độc lập, hành vi bảo vệ nạn nhân là biến phụ thuộc, cho thấy xấp xỉ 19\% sự thay đổi trong hành vi bảo vệ nạn nhân của học sinh chứng kiến có thể được giải thích bởi khu vực cơ chế làm giảm trách nhiệm cá nhân $\left(R^{2}=0,19\right)$. Hệ số hồi quy $\mathrm{B}=-0,43$ $(t=-13,08 ; p<0,05)$ cho thấy khu vực cơ chế này có khả năng dự báo hành vi bảo vệ nạn nhân của học sinh khi chứng kiến bắt nạt. Cụ thể, khi cá nhân ít sử dụng những cơ chế buông lỏng để di chuyển hoặc chia mỏng trách nhiệm can thiệp bắt nạt cho người khác, cá nhân sẽ có xu hướng thực hiện hành động bảo vệ nạn nhân nhiều hơn. Bên cạnh đó, $F_{1 ; 734}=171,11(p<0,05)$ cho thấy kết quả này có thể suy rộng và áp dụng được cho tổng thể với độ tin cậy $99 \%$. 
Hành vi củng cố bắt nat: Kết quả phân tích hồi quy với biến độc lập là khu vực cơ chế làm giảm trách nhiệm cá nhân, biến phụ thuộc là hành vi củng cố bắt nạt, cho thấy $16 \%$ sự thay đổi trong hành vi củng cố bắt nạt của bên chứng kiến có thể được giải thích bằng khu vực cơ chế làm giảm trách nhiệm cá nhân $\left(R^{2}=0,16\right)$. Hệ số hồi quy $\mathrm{B}=0,27$ $(t=11,98 ; p<0,05)$ cho thấy khu vực cơ chế này có khả năng dự báo hành vi củng cố bắt nạt của cá nhân chứng kiến bắt nạt. Cụ thể, khi học sinh có xu hướng sử dụng những cơ chế thuộc khu vực làm giảm trách nhiệm cá nhân để giải thích cho lựa chọn hành vi của mình khi chứng kiến bắt nạt, các em sẽ có xu hướng thực hiện hành vi củng cố, cổ vũ bắt nạt nhiều hơn. Bên cạnh đó, $F_{1 ; 734}=143,59(p<0,05)$ cho thấy kết quả này có thể được suy rộng và áp dụng cho tổng thể với độ tin cậy $99 \%$.

Hành vi không can dư: Kết quả phân tích hồi quy với biến độc lập là khu vực cơ chế làm giảm trách nhiệm cá nhân, biến phụ thuộc là hành vi không can dự của học sinh chứng kiến bắt nạt, cho thấy khu vực cơ chế này có thể giải thích được $14 \%$ sự thay đổi trong hành vi không can dự của học sinh chứng kiến $\left(R^{2}=0,14\right)$. Đồng thời, khu vực làm giảm trách nhiệm cá nhân có khả năng dự báo hành vi không can dự của học sinh chứng kiến bắt nạt ( $B=0,38 ; t=11,11 ; p<0,05)$. Cụ thể, khi học sinh chứng kiến càng sử dụng những cơ chế thuộc khu vực này trong quá trình quyết định hành động của mình tới vụ bắt nạt, các em sẽ có xu hướng lựa chọn việc đứng ngoài cuộc, không can thiệp vào vụ việc. Kết quả này có thể được suy rộng và áp dụng cho tổng thể với độ tin cậy $99 \%\left(F_{1 ; 734}=123,37 ; p<0,05\right)$.

Bảng 2. Kết quả phân tích hồi quy

\begin{tabular}{|c|c|c|c|c|}
\hline & \multicolumn{4}{|c|}{ Làm giảm trách nhiệm cá nhân } \\
\hline & $\mathrm{R}^{2}$ & $\mathrm{~F}$ & $\mathrm{~B}$ & $\mathrm{t}$ \\
\hline $\begin{array}{c}\text { Bảo vệ } \\
\text { nạn nhân }\end{array}$ & 0,19 & $171,11^{* *}$ & $-0,43$ & $-13,08^{* *}$ \\
\hline $\begin{array}{c}\text { Củng cố } \\
\text { bắt nạt }\end{array}$ & 0,16 & $143,59^{* *}$ & 0,27 & $11,98^{* *}$ \\
\hline $\begin{array}{c}\text { Không } \\
\text { can dự }\end{array}$ & 0,14 & $123,37^{* *}$ & 0,38 & $11,11^{* *}$ \\
\hline
\end{tabular}

Ghi chú: ${ }^{* *} p<0,01$.

\section{Bàn luận}

Nghiên cứu hiện tại đã cung cấp một góc nhìn mới về việc quá trình nhận thức đạo đức có thể tham gia tác động, chi phối tới lựa chọn biểu hiện hành vi của học sinh khi chứng kiến bắt nạt như thế nào. Bằng việc xem xét buông lỏng đạo đức theo bốn khu vực cơ chế thay vì là một cấu trúc đơn chiều kích, nghiên cứu đã góp phần làm sáng tỏ về mối quan hệ cụ thể giữa những khu vực cơ chế và hành vi củng cố bắt nạt, bảo vệ nạn nhân, không can dự trong bắt nạt của bên chứng kiến. Về cơ bản, có thể nhận định cá nhân có mức độ buông lỏng đạo đức cao trong bắt nạt sẽ có xu hướng tham gia củng cố bắt nạt và/hoặc lựa chọn biểu hiện hành vi không can dự khi chứng kiến nhiều hơn so với những học sinh khác. Tuy nhiên, không phải mọi khu vực cơ chế của quá trình này sẽ được sử dụng trong việc đưa ra quyết định thực hiện hành động củng cố bắt nạt của học sinh. Kết quả của nghiên cứu cần được nhìn nhận và luận giải đồng thời cùng với hạn chế của nó. Thứ nhất, với hạn chế của nghiên cứu cắt ngang, chúng tôi chưa thể đưa ra những kết luận về chiều hướng thay đổi của buông lỏng đạo đức qua các giai đoạn lứa tuổi của cá nhân. Như vậy, mối quan hệ giữa biến số này và hành vi gây hấn, bắt nạt của thanh thiếu niên nói chung và lựa chọn hành vi của cá nhân khi chứng kiến bắt nạt nói riêng cần phải được tiếp tục khám phá trong những nghiên cứu theo thời gian. Thứ hai, nghiên cứu sử dụng những công cụ đo tự đánh giá, vì vậy câu trả lời của học sinh hoàn toàn có thể chịu sự chi phối bởi mong muốn và kỳ vọng xã hội. Bên cạnh đó, mối quan hệ giữa các khu vực cơ chế và các dạng biểu hiện tác động trong những dạng bắt nạt khác nhau cần tiếp tục được làm rõ, bởi đặc trưng của từng dạng bắt nạt (trực tiếp và gián tiếp) có thể có những ảnh hưởng nhất định tới quá trình nhận thức và lập luận đạo đức của cá nhân. Quá trình buông lỏng đạo đức có thể sẽ chỉ diễn ra trong một số trường hợp, tình huống đặc thù thay vì là trong mọi bối cảnh (Bandura, 2002) [29]. Đồng thời, xuất phát từ tính chất nhóm của hiện tượng bắt nạt (O’Connell và cộng sự, 1999) [4], những yếu tố thuộc tâm lý nhóm, bao gồm 
chuẩn mực nhóm, không khí lớp học cũng cần được xem xét về khả năng và mức độ ảnh hưởng tới quá trình nhận thức đạo đức của cá nhân.

Kết quả của nghiên cứu này đã cung cấp thêm chất liệu cho những người thực hành tâm lý học/tâm lý học trường học, giáo dục học trong công tác phòng ngừa, can thiệp bắt nạt học đường. Cùng với việc hướng dẫn kỹ năng, kỹ thuật ứng phó và định hướng hành vi thuận xã hội, những chiến lược với mục tiêu làm giảm tần suất sử dụng các cơ chế buông lỏng đạo đức và nâng cao ý thức trách nhiệm cá nhân của học sinh nên được cân nhắc triển khai như một nội dung trong chương trình phòng ngừa bắt nạt toàn trường và toàn khối. Việc giới thiệu và tạo điều kiện cho học sinh thảo luận về những tình huống xuất hiện buông lỏng đạo đức có thể giúp học sinh nhận biết được khi nào những lập luận và hành vi buông lỏng đạo đức được sử dụng trong tình huống bắt nạt, từ đó giúp các em hạn chế sử dụng những cơ chế buông lỏng và có xu hướng thực hiện hành vi bảo vệ, giúp đỡ nạn nhân nhiều hơn. Bên cạnh đó, việc triển khai đồng thời những chương trình phát triển năng lực cảm xúc-xã hội với mục tiêu nâng cao trách nhiệm cá nhân cũng có thể góp phần vào giảm thiểu hành vi ủng hộ, cổ xúy bắt nạt và giúp học sinh nhận diện được vị trí tích cực của mình trong việc hỗ trợ nạn nhân, can thiệp bắt nạt bằng biện pháp ôn hòa.

\section{Kết luận}

Kết quả nghiên cứu trên 736 học sinh THCS cho thấy có sự khác biệt giữa hai giới trong khu vực cơ chế phủ nhận/bóp méo hậu quả. Đồng thời, kết quả cũng cho thấy có sự khác biệt ở việc sử dụng những khu vực cơ chế buông lỏng đạo đức trong bắt nạt giữa các khối lớp, cụ thể: học sinh khối 8 thể hiện rõ hơn học sinh khối 9 ở khu vực cơ chế quy kết trách nhiệm cho nạn nhân; học sinh khối 6 thể hiện ít hơn học sinh ba khối còn lại ở khu vực cơ chế phủ nhận/bóp méo hậu quả và làm giảm trách nhiệm cá nhân; học sinh khối 8 thể hiện rõ hơn học sinh khối 7 và khối 9 ở khu vực cơ chế làm giảm trách nhiệm cá nhân. Sự khác biệt giữa hai giới và các khối lớp này có thể được xem là gợi mở về ảnh hưởng của những yếu tố nhân khẩu học với quá trình buông lỏng đạo đức, cũng như chiều hướng thay đổi của quá trình này theo các giai đoạn lứa tuổi của cá nhân.

Như kỳ vọng ban đầu của nghiên cứu, cả bốn khu vực cơ chế buông lỏng đạo đức có tương quan nghịch với hành vi bảo vệ nạn nhân, tương quan thuận với hành vi không can dự. Tuy nhiên, chỉ có ba khu vực cơ chế có tương quan nghịch có ý nghĩa với hành vi củng cố bắt nạt, bao gồm: làm giảm trách nhiệm cá nhân, phủ nhận/bóp méo hậu quả, và quy kết trách nhiệm cho nạn nhân. Kết quả này cho thấy lựa chọn hành vi của học sinh chứng kiến trước các tình huống bắt nạt có liên quan tới quá trình nhận thức đạo đức của cá nhân.

Kết quả phân tích hồi quy cho thấy khu vực cơ chế làm giảm trách nhiệm cá nhân có thể được xem như một yếu tố có khả năng dự báo các biểu hiện hành vi khác nhau của bên chứng kiến trong bắt nạt. Cụ thể, khi học sinh lập luận để xóa bỏ hoặc làm giảm trách nhiệm cá nhân với hành động của bản thân càng nhiều, cá nhân sẽ có $\mathrm{xu}$ hướng thực hiện hành vi củng cố bắt nạt và không can dự. Ngược lại, khi học sinh ít sử dụng cơ chế để di chuyển trách nhiệm và chia mỏng trách nhiệm, các em sẽ có xu hướng thực hiện những hành động mang tính chất bảo vệ, hỗ trợ nạn nhân như cố gắng làm bạn và/hoặc báo cho người lớn. Mối quan hệ này có thể được giải thích bằng hiệu ứng bên chứng kiến cổ điển (Darley và Latané, 1968; Salmivalli, 2010) [41, 42], khi càng nhiều người chứng kiến một tình huống khẩn cấp cần can thiệp, cá nhân chứng kiến càng ít có động lực, ít nhận thấy mình có trách nhiệm cần can thiệp, giúp đỡ nạn nhân vì họ kỳ vọng những người khác đang cùng chứng kiến sẽ làm điều đó. Như vậy, nếu như cá nhân học sinh ít có xu hướng sử dụng những cơ chế để di chuyển, chia mỏng trách nhiệm can thiệp bắt nạt cho những người khác, cá nhân có thể sẽ xem xét và nhận định bản thân mình có vai trò, trách nhiệm trong việc giúp đỡ nạn nhân bắt nạt và can thiệp vụ bắt nạt, từ đó kỳ vọng sẽ thực hiện những hành động cụ thể để bảo vệ nạn nhân. 


\section{Tài liệu tham khảo}

[1] D. Olweus, Bullying at School, in Aggressive Behavior, Springer, 1994, pp. 97-130.

[2] W. M. Craig, D. Pepler, R. Atlas, Observations of Bullying in the Playground and in the Classroom, School Psychology International, Vol. 21, No. 1, 2000, pp. 22-36, https://doi.org/10.1177/0143034300211002.

[3] D. Hawkins, D. Pepler, W. Craig, Naturalistic Observations of Peer Interventions in Bullying, Social Development, Vol. 10, 2001, pp. 512-527, https://doi.org/10.1111/1467-9507.00178.

[4] P. O'Connell, D. Pepler, W. Craig, Peer Involvement in Bullying: Insights and Challenges for Intervention, Journal of Adolescence, Vol. 22, No. 4), 1999, pp. 437-452, https://doi.org/10.1006/jado.1999.0238.

[5] K. Rigby, B. Johnson, Expressed Readiness of Australian Schoolchildren to Act as Bystanders in Support of Children Who are Being Bullied, Educational Psychology, Vol. 26, No. 3, 2006, pp. $425-440$.

[6] C. Salmivalli, K. Lagerspetz, K. Björkqvist, K. Österman, A. Kaukiainen, Bullying as a Group Process: Participant Roles and Their Relations to Social Status Within the Group, Aggressive Behavior: Official Journal of the International Society for Research on Aggression, Vol. 22, No. 1, 1996, pp. 1-15.

[7] C. Salmivalli, R. Voeten, Connections Between Attitudes, Group Norms, and Behaviour in Bullying Situations, International Journal of Behavioral Development, Vol. 28, 2004, pp. 246-258, https://doi.org/10.1080/01650250344000488,

[8] C. Salmivalli, Participant Role Approach to School Bullying: Implications for Interventions, Journal of Adolescence, Vol. 22, No. 4, 1999, pp. 453-459, https://doi.org/10.1006/jado.1999.0239.

[9] C. Salmivalli, Participant Roles in Bullying: How Can Peer Bystanders Be Utilized in Interventions?, Theory Into Practice, Vol. 53, No. 4, 2014, pp. 286-292, https://doi.org/10.1080/00405841.2014.947222.

[10] N. Vannini, S. Enz, M. Sapouna, D. Wolke, S. Watson, S. Woods, K. Dautenhahn, L. Hall, A. Paiva, E. André, R. Aylett, W. Schneider, FearNot!: A Computer-based Anti-bullying-Programme Designed to Foster Peer Intervention, European Journal of Psychology of Education, Vol. 26, No. 1, 2011, pp. 21-44, https://doi.org/10.1007/s10212-010-0035-4.

[11] R. Thornberg, A Student in Distress: Moral Frames and Bystander Behavior in School, The Elementary School Journal, Vol. 110, No. 4, 2010, pp. 585-608.
[12] K. Barchia, K. Bussey, Predictors of Student Defenders of Peer Aggression Victims: Empathy and Social Cognitive Factors, International Journal of Behavioral Development, Vol. 35, No. 4, 2011, pp. 289-297.

[13] M. Bjärehead, R. Thornberg, L. Wänström, G. Gini, Mechanisms of Moral Disengagement and Their Associations with Indirect Bullying, Direct Bullying, and Pro-aggressive Bystander Behavior, The Journal of Early Adolescence, Vol. 40, No. 1, 2020, pp. 28-55.

[14] G. Gini, Social Cognition and Moral Cognition in Bullying: What's Wrong?, Aggressive Behavior: Official Journal of the International Society for Research on Aggression, Vol. 32, No. 6, 2006, pp. 528-539.

[15] S. Hymel, N. Rocke-Henderson, R. A. Bonanno, Moral Disengagement: A Framework for Understanding Bullying Among Adolescents, Journal of Social Sciences, Vol. 8, No. 1, 2005, pp. 1-11.

[16] R. Thornberg, T. Jungert, Bystander Behavior in Bullying Situations: Basic Moral Sensitivity, Moral Disengagement and Defender Self-efficacy, Journal of Adolescence, Vol. 36, No. 3, 2013, pp. 475-483, https://doi.org/10.1016/j.adolescence.2013.02.003.

[17] R. Thornberg, T. Jungert, School Bullying and the Mechanisms of Moral Disengagement, Aggressive Behavior, Vol. 40, No. 2, 2014, pp. 99-108.

[18] R. Thornberg, L. Wänström, T. Pozzoli, Peer Victimisation and Its Relation to Class Relational Climate and Class Moral Disengagement Among School Children, Educational Psychology, Vol. 37, No. 5, 2017, pp. 524-536, https://doi.org/10.1080/01443410.2016.1150423.

[19] T. V. Cong, The Status of Peer Victimization among Vietnamese School Students, VNU Journal of Social Sciences and Humanities, Vol. 3, No. 4, 2017, pp. 465-479.

[20] T. V. Cong, Bullying Among Students in the Digital Age, Journal of Psychology - Vietnam Academy of Social Sciences, Vol. 11, No. 236, 2018, pp. 28-41.

[21] T. V. Cong, B. Weiss, D. Cole, Peer Victimization and Its Relation with Self-perception, Depression Among High School Students, Journal of Psychology - Vietnam Academy of Social Sciences, Vol. 11, No. 128, 2009, pp. 50-59.

[22] H. T. H. Le, M. P. Dunne, M. A. Campbell, M. L. Gatton, H. T. Nguyen, N. T. Tran, Temporal Patterns and Predictors of Bullying Roles Among Adolescents in Vietnam: A school-based Cohort Study, Psychology, Health \& Medicine, Vol. 22, No. 1, 2017, pp. 107-121, https://doi.org/10.1080/13548506.2016.1271953. 
[23] P. T. T. Ba, T. Q. Anh, Cyberbullying Among High School Students and some Related Factors, Journal of Medical Research - Hanoi Medical University, Vol. 104, No. 6, 2016, pp. 35-42.

[24] T. V. Cong, N. T. H. Phuong, The Relation Between Cyberbullying and Friendship Quality Among Highschool Students in Hanoi, Journal of Science and Technology, Vol. 60, No. 4, 2018, pp. 1-5.

[25] C. V. Tran, B. Weiss, N. P. H. Nguyen, Academic Achievement, and Cyber-bullying and Cyber-victimization Among Middle-and high-school Students in Vietnam, International Journal of School \& Educational Psychology, 2020, pp. 1-10, https://doi.org/10.1080/21683603.2020.1837700.

[26] C. V. Tran, N. P. H. Nguyen, B. Weiss, L. V. Nguyen, D. B. Nguyen, Definition and Characteristics of "Cyberbullying" Among Vietnamese Students, VNU Journal of Science: Education Research, Vol. 34, No. 4, 2018, pp. 1-10, https://doi.org/10.25073/2588-1159/vnuer.4212.

[27] A. Bandura, C. Barbaranelli, G. V. Caprara, C. Pastorelli, Mechanisms of Moral Disengagement in the Exercise of Moral Agency, Journal of Personality and Social Psychology, Vol. 71, No. 2, 1996, 364-374.

[28] A. Bandura, Moral Disengagement in the Perpetration of Inhumanities, Personality and Social Psychology Review, Vol. 3, No. 3, 1999, pp. 193-209.

[29] A. Bandura, Selective Moral Disengagement in the Exercise of Moral Agency, Journal of Moral Education, Vol. 31, No. 2, 2002, pp. 101-119.

[30] A. Bandura, Social Foundations of Thought and Action: A Social Cognitive Theory, Englewood Cliffs, 1986.

[31] A. Bandura, Social Cognitive Theory of Moral Thought and Action, Psychology Press, 2014.

[32] A. Bandura, Moral Disengagement: How People do Harm and Live with Themselves, Worth Publishers, 2016.

[33] M. J. Boulton, K. Underwood, Bully/victim Problems Among Middle School Children, British Journal of Educational Psychology, Vol. 62, No. 1, 1992, pp. 73-87.
[34] C. D. Pornari, J. Wood, Peer and Cyber Aggression in Secondary School Students: The Role of Moral Disengagement, Hostile Attribution Bias, and Outcome Expectancies, Aggressive Behavior: Official Journal of the International Society for Research on Aggression, Vol. 36, No. 2, 2010, pp. 81-94.

[35] S. Perren, E. Gutzwiller-Helfenfinger, Cyberbullying and Traditional Bullying in Adolescence: Differential Roles of Moral Disengagement, Moral Emotions, and Moral Values, European Journal of Developmental Psychology, Vol. 9, No. 2, 2012, pp. 195-209.

[36] K. Bussey, A. Luo, S. Fitzpatrick, K. Allison, Defending Victims of Cyberbullying: The Role of Self-efficacy and Moral Disengagemen, Journal of School Psychology, Vol. 78, 2020, pp. 1-12.

[37] S. C. Caravita, J. J. Sijtsema, J. A. Rambaran, G. Gini, Peer Influences on Moral Disengagement in Late Childhood and Early Adolescence, Journal of Youth and Adolescence, Vol. 43, No. 2, 2014, pp. 193-207.

[38] G. Gini, T. Pozzoli, K. Bussey, The Role of Individual and Collective Moral Disengagement in Peer Aggression and Bystanding: A Multilevel Analysis, Journal of Abnormal Child Psychology, Vol. 43, No. 3, 2015, pp. 441-452.

[39] M. L. Obermann, Moral Disengagement Among Bystanders to School Bullying, Journal of School Violence, Vol. 10, No. 3, 2011, pp. 239-257, https://doi.org/10.1080/15388220.2011.578276.

[40] S. C. Caravita, G. Gini, T. Pozzoli, Main and Moderated Effects of Moral Cognition and Status on Bullying and Defending, Aggressive Behavior, Vol. 38, No. 6, 2012, pp. 456-468.

[41] J. M. Darley, B. Latané, Bystander Intervention in Emergencies: Diffusion of Responsibility, Journal of Personality and Social Psychology, Vol. 8, No. 4, Part 1, 1968, pp. 377-383.

[42] C. Salmivalli, Bullying and the Peer Group: A Review, Special Issue on Group Processes and Aggression, Vol. 15, No. 2, 2010, pp. 112-120, https://doi.org/10.1016/j.avb.2009.08.007. 